\title{
From Masked Language Modeling to Translation: Non-English Auxiliary Tasks Improve Zero-shot Spoken Language Understanding
}

\author{
Rob van der Goot $^{1}$, Ibrahim Sharaf ${ }^{2}$, Aizhan Imankulova ${ }^{3}$, Ahmet Üstün ${ }^{4}$, \\ Marija Stepanovic $^{1}$, Alan Ramponi ${ }^{5,6}$, Siti Oryza Khairunnisa ${ }^{3}$, Mamoru Komachi ${ }^{3}$, \\ Barbara Plank ${ }^{1}$ \\ ${ }^{1}$ IT University of Copenhagen, ${ }^{2}$ Factmata, ${ }^{3}$ Tokyo Metropolitan University, ${ }^{4}$ University of \\ Groningen, ${ }^{5}$ University of Trento, ${ }^{6}$ Fondazione The Microsoft Research - University of Trento \\ Centre for Computational and Systems Biology (COSBI) \\ $\{$ robv, bapl\}aitu.dk
}

\begin{abstract}
The lack of publicly available evaluation data for low-resource languages limits progress in Spoken Language Understanding (SLU). As key tasks like intent classification and slot filling require abundant training data, it is desirable to reuse existing data in high-resource languages to develop models for low-resource scenarios. We introduce XSID, a new benchmark for cross-lingual (X) Slot and Intent Detection in 13 languages from 6 language families, including a very low-resource dialect. To tackle the challenge, we propose a joint learning approach, with English SLU training data and non-English auxiliary tasks from raw text, syntax and translation for transfer. We study two setups which differ by type and language coverage of the pre-trained embeddings. Our results show that jointly learning the main tasks with masked language modeling is effective for slots, while machine translation transfer works best for intent classification. ${ }^{1}$
\end{abstract}

\section{Introduction}

Digital conversational assistants have become an integral part of everyday life and they are available, e.g., as standalone smart home devices or in smartphones. Key steps in such task-oriented conversational systems are recognizing the intent of a user's utterance, and detecting the main arguments, also called slots. For example, for an utterance like "Add reminder to swim at 11 am tomorrow", these key Natural Language Understanding (NLU), or Spoken Language Understanding (SLU) tasks are illustrated in Figure 1. As slots depend on the intent type, leading models typically adopt joint solutions (Chen et al., 2019; Qin et al., 2020).

Despite advances in neural modeling for slot and intent detection ( $(6)$, datasets for SLU remain limited, hampering progress toward providing SLU for many language varieties. Most avail-

\footnotetext{
${ }^{1}$ The source code, dataset and predictions are available at: https://bitbucket.org/robvanderg/xsid
}

Add reminder to swim at 11 am tomorrow
intent: add_reminder

Figure 1: English example from XSID annotated with intents (add_reminder) and slots (todo, datetime). The full set of languages is shown in Table 2.

able datasets either support only a specific domain (like air traffic systems) (Xu et al., 2020), or are broader but limited to English and a few other languages (Schuster et al., 2019; Coucke et al., 2018). We release XSID, a new benchmark intended for SLU evaluation in low-resource scenarios. XSID contains evaluation data for 13 languages from six language families, including a very low-resource dialect. It homogenizes annotation styles of two recent datasets (Schuster et al., 2019; Coucke et al., 2018) and provides the broadest public multilingual evaluation data for modern digital assistants.

Most previous efforts to multilingual SLU typically focus on translation or multilingual embeddings transfer. In this work, we propose an orthogonal approach, and study non-English auxiliary tasks for transfer. We hypothesize that jointly training on target language auxiliary tasks helps to learn properties of the target language while learning a related task simultaneously. We expect that this helps to refine the multilingual representations for better SLU transfer to a new language. We evaluate a broad range of auxiliary tasks not studied before in such combination, exploiting raw data, syntax in Universal Dependencies (UD) and parallel data.

Our contributions i) We provide XSID, a new cross-lingual SLU evaluation dataset covering Arabic (ar), Chinese (zh), Danish (da), Dutch (nl), English (en), German (de), Indonesian (id), Italian (it), Japanese (ja), Kazakh (kk), Serbian (sr), Turkish (tr) and an Austro-Bavarian German dialect, South Tyrolean (de-st). ii) We experiment with new 


\begin{tabular}{|c|c|c|c|c|c|c|c|}
\hline Dataset & Source & Langs. & Lang. Fams. & Domains $^{2}$ & Intents & Slots & \#sents \\
\hline Atis & Hemphill et al. (1990) & 1 & 1 & 1 & 24 & 83 & 5,871 \\
\hline Snips & Coucke et al. (2018) & 1 & 1 & 5 & 7 & 39 & 14,484 \\
\hline HWU64 & Xingkun Liu and Rieser (2019) & 1 & 1 & 22 & 64 & 54 & 25,716 \\
\hline Almawave-SLU & Bellomaria et al. (2019) & 1 & 1 & 1 & 7 & 39 & 8,542 \\
\hline $\mathrm{CSTOP}^{\dagger}$ & Einolghozati et al. (2021) & 2 & 1 & 2 & 19 & 10 & 5,800 \\
\hline Leyzer* & Sowański and Janicki (2020) & 3 & 1 & 20 & 186 & 86 & 16,257 \\
\hline Facebook & Schuster et al. (2019) & 3 & 2 & 3 & 12 & 11 & 57,049 \\
\hline multiAtis++ & Xu et al. (2020) & 9 & 4 & 1 & 23 & 83 & 45,046 \\
\hline XSID & This work & 13 & 6 & 7 & 16 & 33 & 10,000 \\
\hline
\end{tabular}

Table 1: Existing SLU datasets. Note that XSID is intended to serve as evaluation data only (Snips+Facebook can be used as English training data). ${ }^{\dagger}$ Code-switched data (Spanglish). ${ }^{*}$ Automatically generated data. Language families are counted based on highest level of Glottolog (Hammarström and Nordhoff, 2011).

non-English auxiliary tasks for joint cross-lingual transfer on slots and intents: UD parsing, machine translation (MT), and masked language modeling. iii) We compare our proposed models to strong baselines, based on multilingual pre-trained language models mBERT (Devlin et al., 2019) and xlm-mlm-tlm-xnli15-1024 (Conneau et al., 2020) (henceforth XLM15), where the former was pre-trained on 12 of our 13 languages, and XLM15 on 5 of our 13 languages, thereby simulating a lowresource scenario. We also compare to a strong machine translation model (Qin et al., 2020).

The remainder of this paper is structured as follows: we start by giving an overview of existing datasets and introduce XSID $(\S 2)$, then we discuss our baselines and proposed extensions (§ 3). After this, we discuss the performance of these models (§ 4), and provide an analysis (§ 5) before we end with the related work on cross-lingual SLU (§ 6) and the conclusion (§7).

\section{Data}

\subsection{Other SLU Datasets}

An overview of existing datasets is shown in Table 1 . It should be noted that we started the creation of XSID at the end of 2019, when less variety was available. We choose to use the Snips (Coucke et al., 2018) and Facebook (Schuster et al., 2019) data as a starting point.

Most existing datasets are English only (all datasets in Table 1 include English), and they differ in the domains they cover. For example, Atis (Hemphill et al., 1990) is focused on airlinerelated queries, CSTOP (Einolghozati et al., 2021)

\footnotetext{
${ }^{2}$ The notion of domain is ill-defined within the scope of this task. We report the numbers from the paper, and, for Snips, we have identified the following: alarm, reminder, weather, restaurant, creative works.
}

contains queries about wheather and devices, and other datasets cover multiple domains.

Extensions of Atis to new languages are a main direction. These include translations to Chinese (He et al., 2013), Italian (Bellomaria et al., 2019), Hindi and Turkish (Upadhyay et al., 2018) and very recently, the MultiAtis++ corpus (Xu et al., 2020) with 9 languages in 4 language families. To the best of our knowledge, this is the broadest publicly available SLU corpus to date in terms of the number of languages, yet the data itself is less varied. Almost simultaneously, Schuster et al. (2019) provide a dataset for three new topics (alarm, reminder, weather) in three languages (English, Spanish and Thai). English utterances for a given intent were first solicited from the crowd, translated into two languages (Spanish and Thai), and manually annotated for slots. We follow these approaches, but depart from the Snips (Coucke et al., 2018) and Facebook (Schuster et al., 2019) datasets to create a more varied resource covering 13 languages, while homogenizing the annotations.

XSID is a cross-lingual SLU evaluation dataset covering 13 languages from six language families with English training data. In what follows, we provide details on the creation of XSID ( $\$ 2.2$ ), including homogenization of annotation guidelines and English source training data ( $\$ 2.3)$. For data statement and guidelines, we refer the reader to Section E, F and G in the Appendix.

\subsection{XSID}

As a starting point, we extract 400 random English utterances from the Snips data (Coucke et al., 2018) as well as 400 from the Facebook data (Schuster et al., 2019), which for both consist of 250 utterances from the test-split and 150 from the dev-split. We maintain the splits from the original data in 


\begin{tabular}{|c|c|c|}
\hline Lang. & Language Family & Annotation \\
\hline ar & Afro-Asiatic & أود أن أرى مواعيد عرض فيلم Silly Movie 2.0 في دار السينما \\
\hline da & Indo-European & Jeg vil gerne se spilletiderne for Silly Movie 2.0 i biografen \\
\hline de & Indo-European & Ich würde gerne den Vorstellungsbeginn für Silly Movie 2.0 im Kino sehen \\
\hline de-st & Indo-European & I mecht es Programm fir Silly Movie 2.0 in Film Haus sechn \\
\hline en & Indo-European & I'd like to see the showtimes for Silly Movie 2.0 at the movie house \\
\hline id & Austronesian & Saya ingin melihat jam tayang untuk Silly Movie 2.0 di gedung bioskop \\
\hline it & Indo-European & Mi piacerebbe vedere gli orari degli spettacoli per Silly Movie 2.0 al cinema \\
\hline ja & Japonic & 映画館 の Silly Movie 2.0 の上映時間を見せて。 \\
\hline $\mathrm{kk}$ & Turkic & Мен Silly Movie 2.0 бағдарламасының кинотеатрда көрсетілім уақытын көргім келеді \\
\hline $\mathrm{nl}$ & Indo-European & Ik wil graag de speeltijden van Silly Movie 2.0 in het filmhuis zien \\
\hline sr & Indo-European & Želela bih da vidim raspored prikazivanja za Silly Movie 2.0 u bioskopu \\
\hline $\operatorname{tr}$ & Turkic & Silly Movie 2.0'ın sinema salonundaki seanslarını görmek istiyorum \\
\hline $\mathrm{zh}$ & Sino-Tibetan & 我想看 Silly Movie 2.0 在 影院 的放映 \\
\hline
\end{tabular}

Table 2: Examples of annotation for all languages in our dataset with intent: SearchScreeningEvent, and two slots: movie_name and object_location_type. Includes information on language families from Glottolog (Hammarström and Nordhoff, 2011).

XSID (i.e. sentences in XSID test are from Snips test or Facebook test). We then translate this sample into all of our target languages. It should be noted that some duplicates occur in the random sample of the Facebook data. Since these instances naturally occur more often, we decided to retain them to give a higher weight to common queries in the final evaluation. ${ }^{3}$

XSID includes Arabic (ar), Chinese (zh), Danish (da), Dutch (nl), English (en), German (de), Indonesian (id), Italian (it), Japanese (ja), Kazakh (kk), Serbian (sr), Turkish (tr) and an Austro-Bavarian German dialect, South Tyrolean (de-st). ${ }^{4}$ We have 13 evaluation languages with 800 sentences per language ${ }^{5}$ resulting in a final dataset of 10,000 sentences. The language selection is based on availability of translators/annotators (most of them are co-authors of this paper, i.e. highly-educated with a background in NLP). We favor this setup over crowd-sourcing, i.e. quality and breadth in annotation and languages, and because for some languages crowd-sourcing is not an option. ${ }^{6}$ For more information on the data and annotators we refer to the dataset statement in Appendix E.

\footnotetext{
${ }^{3}$ This decision has been made after discussion with a realworld digital assistant team.

${ }^{4}$ The dialect is spoken by roughly 450,000 speakers in an Alpine province in Northern Italy. It has no official ISO language code nor a normed writing form.

${ }^{5}$ Except for Japanese where we only have the Facebook data.

${ }^{6} \mathrm{We}$ did not have access to native speakers of Thai and Spanish part of the Facebook data (Schuster et al., 2019), which is why there are not included (yet).
}

The first step of the dataset creation was the translation. For this, the goal was to provide a fluent translation which was as close as possible to the original meaning. Because the data consists of simple, short utterances, we consider our annotator pool to be adequate for this task (even though they are not professional translators). The intents could easily be transferred from the English data, but the slots needed to be re-annotated, which was done by the same annotators.

Unfortunately, we were unable to retrieve annotation guidelines from the earlier efforts. Hence, as a first step of and as part of training, we derived annotation guidelines by jointly re-annotating dev and test portions of the English parts of the two data sources. These guidelines were revised multiple times in the process to derive the final guidelines for the whole dataset. Ultimately, the data collection process proceeded in two steps: translation of the data from English, and slot annotation in the target language. The aim of the guidelines was to generalize labels to make them more broadly applicable to other intent subtypes, and remove within-corpus annotation variation (see Appendix $\mathrm{G}$ for details). We calculated inter-annotator agreement for the guidelines; three annotators native in Dutch annotated 100 samples, and reached a Fleiss Kappa (Fleiss, 1971) score of 0.924 , which is very high agreement. Common mistakes included annotation of question words, inclusion of locations in reminders, and the inclusion of function words in the spans. We updated the guidelines after the agreement study. 
After these target phase annotation rounds, we finalized the guidelines, which are provided in the Appendix $G$ and form the basis for the provided data. Table 2 provides an example annotation for all 13 languages for the example sentence "I'd like to see the showtimes for Silly Movie 2.0 at the movie house". These example translations illustrate not only the differences in scripts, but also differences in word order and length of spans, confirming the distances between the languages.

\subsection{English Training Data}

Because of our revised guidelines for the Facebook data and mismatches in granularity of labels between the Snips and Facebook data, we homogenize the original training data for both sources and include it in our release. For the Facebook data, this includes rule-based fixing of spans and recognition of the REFERENCE and RECURRING_TIME labels. ${ }^{7}$ For the Snips data, we convert a variety of labels that describe a location to the LOCATION label which is used in the Facebook data, and labels describing a point or range in time to DATETIME. After this process, we simply concatenate both resulting datasets, and shuffle them before training. The resulting training data has 43,605 sentences.

\section{Models}

Our main hypothesis is that we can improve zeroshot transfer with target-language auxiliary tasks. We hypothesize that this will help the multilingual pre-trained base model to learn peculiarities about the target language, while it is learning the target task as well. To this end, we use three (sets of) tasks with a varying degree of complexity and availability: 1) Masked Language Modeling (MLM): which is in spirit similar to pre-training on another domain (Gururangan et al., 2020), however, we learn this jointly with the target task to avoid catastrophic forgetting (McCloskey and Cohen, 1989); 2) Neural Machine Translation (NMT): where we learn English SLU as well as translation from English to the target language; and 3) Universal Dependency (UD) parsing: to insert linguistic knowledge into the shared parameter space to learn from syntax as auxiliary task besides learning the SLU task.

In the following subsections, we first describe the implementation of our baseline model, and the machine translation-based model, and then de-

\footnotetext{
${ }^{7}$ For more details on this procedure, we refer to scripts/0.fixOrigAnnotation.py in the repo.
}

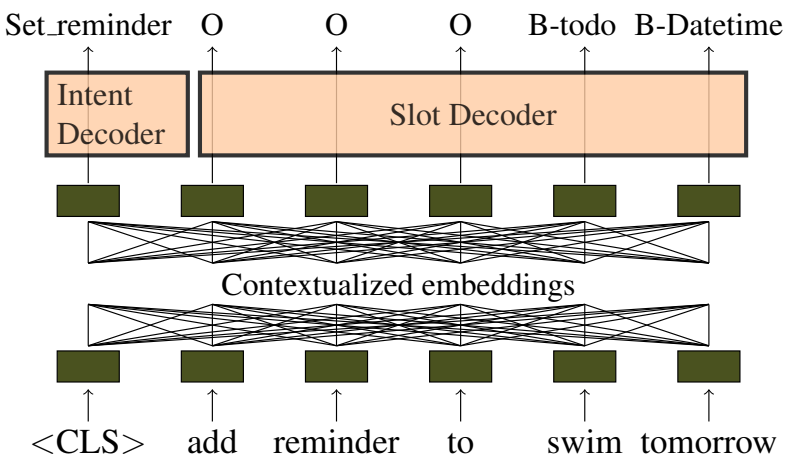

Figure 2: Overview of the baseline model.

scribe the implementation of all auxiliary tasks (and the data used to train them). Auxiliary tasks are sorted by dataset availability (MLM $\succ$ NMT $\succ$ UD), where the first type can be used with any raw text, the second one needs parallel data - which is readily available for many languages as a byproduct of multilingual data sources - and the last one requires explicit human annotation. For South Tyrolean, a German dialect, no labeled target data of any sort is available; we use the German task data instead. We provide more details of data sources and sizes in Appendix B.

\subsection{Baseline}

All our models are implemented in MaChAmp v0.2 (van der Goot et al., 2021), an AllenNLPbased (Gardner et al., 2018) multi-task learning toolkit. It uses contextual embeddings, and finetunes them during training. In the multi-task setup, the encoding is shared, and each task has its own decoder. For slot prediction, a greedy decoding with a softmax layer is used, for intents it uses a linear classification layer over the [CLS] token (see Figure 2). ${ }^{8}$ The data for each task is split in batches, and the batches are then shuffled. We use the default hyperparameters of MaChAmp for all experiments which were optimized on a wide variety of tasks (van der Goot et al., 2021). ${ }^{9}$ The following models are extensions of this baseline. In the NMT-transfer model ( $\$ 3.2)$, the training data is translated before passing it into the model. For the auxiliary models ( $\$ 3.3,3.4$ and 3.5), we simply add another decoder next to the intent and slot decoders. The losses are summed, and typically weighted (multiplied) by a factor which is given in

\footnotetext{
${ }^{8} \mathrm{We}$ also tried to use a CRF layer for slots which consistently led to lower performance.

${ }^{9}$ Hyperparameter settings used in experiments are reported in Appendix A.
} 
corresponding subsections. We enable the proportional sampling option of MaChAmp (multinomial sampling $\alpha=0.5$ ) in all multi-task experiments, to avoid overfitting to the auxiliary task.

\subsection{Neural Machine Translation with Attention (nmt-transfer)}

For comparison, we trained a NMT model to translate the NLU training data into the target language, and map the annotations using attention. As opposed to most previous work using this method (Xu et al., 2020; He et al., 2013; Schuster et al., 2019), we opt for an open-source implementation and provide the scripts to rerun the experiments. More specifically, we use the Fairseq toolkit (Ott et al., 2019) implementation of the Transformer-based model (Vaswani et al., 2017) with default hyperparameters. Sentences were encoded using bytepair encoding (BPE) (Sennrich et al., 2016), with a shared vocabulary of 32,000 tokens. At inference time, we set the beam size to 4 , and extracted alignment scores to target tokens calculated from the attention weights matrix. These scores are used to align annotation labels to target language outputs; we map the label of each token to the highest scoring alignment target token. We convert the output to valid BIO tags: we use the label of the B for the whole span, and an I following an $\mathrm{O}$ is converted to a $\mathrm{B}$.

Data To ensure that our machine translation data is suitable for the target domain, we choose to use a combination of transcribed spoken parallel data. For languages included in the IWSLT 2016 Ted talks dataset (Cettolo et al., 2016), we use the train and development data included, and enlarge the training data with the training split from Opensubtitles $^{10} 2018$ (Lison and Tiedemann, 2016), and Tatoeba (Tiedemann, 2012). For languages absent in IWSLT2016, we used the Opensubtitles data for training and Tatoeba as development set. For Kazakh, the Opensubtitles data only contains 2,000 sentences, so we concatenated out-ofdomain data from the WMT2019 data (Barrault et al., 2019), consisting of English-Kazakh crawled corpora. We adapt the BertBasic tokenizer (which splits punctuation, it does not perform subword tokenization) to match the Facebook and Snips dataset tokenization and use this to pre-tokenize the data.

\footnotetext{
${ }^{10} \mathrm{http}: / / \mathrm{www}$. opensubtitles.org/
}

\subsection{Masked Language Modeling (aux-mlm)}

Previous work has shown that continuing to train a language model with an MLM objective on raw data close to the target domain leads to performance improvements (Gururangan et al., 2020). However, in our setup, task-specific training data and target data are from different languages. Therefore, in order to learn to combine the language and the task in a cross-lingual way, we train the model jointly with MLM and task-specific classification objective on target and training languages respectively. We apply the original BERT masking strategy and we do not include next sentence prediction following Liu et al. (2019a). For computational efficiency, we limit the number of input sentences to 100,000 and use a loss weight of 0.01 for MLM training.

Data For our masked language modeling objective, we use the target language machine translation data described above.

\subsection{Machine Translation (aux-nmt)}

To jointly learn to transfer linguistic knowledge from English to the target language together with the target task, we implement a NMT decoder based on the shared encoder. We use a sequenceto-sequence model (Sutskever et al., 2014) with a recurrent neural network decoder, which suits the auto-regressive nature of the machine translation tasks (Cho et al., 2014), and an attention mechanism to avoid compressing the whole source sentence into a fixed-length vector (Bahdanau et al., 2015). We found that fine-tuning the shared encoder achieves good performance on our machine translation datasets (Conneau and Lample, 2019; Clinchant et al., 2019), alleviating the need for freezing its parameters during training in order to avoid catastrophic forgetting (Imamura and Sumita, 2019; Goodfellow et al., 2014). Similar to MLM, we use 100,000 sentences, and a weight of 0.01 .

Data For this auxiliary task, we use the same data as for NMT-TRANSFER, described in detail above.

\subsection{Universal Dependencies (aux-ud)}

Using syntax in hierarchical multi-task learning has previously shown to be beneficial (Hashimoto et al., 2017; Godwin et al., 2016). We here use full Universal Dependency (UD) parsing, i.e., partof-speech (POS) tagging, lemmatization, morphological tagging and dependency parsing as joint 


\begin{tabular}{|c|c|c|c|c|c|c|c|c|c|c|c|c|c|c|}
\hline $\begin{array}{l}\text { mBERT } \\
\text { lang2vec }\end{array}$ & en & $\frac{\mathrm{de}-\mathrm{st}}{-}$ & $\begin{array}{r}\text { de } \\
0.18\end{array}$ & $\begin{array}{r}\text { da } \\
0.18\end{array}$ & $\begin{array}{r}\text { nl } \\
0.19\end{array}$ & $\begin{array}{r}\text { it } \\
0.22\end{array}$ & $\begin{array}{r}\mathbf{s r} \\
0.23\end{array}$ & $\begin{array}{r}\text { id } \\
0.24\end{array}$ & $\begin{array}{r}\text { ar } \\
0.30\end{array}$ & $\begin{array}{r}\mathbf{z h} \\
0.33\end{array}$ & $\begin{array}{r}\mathbf{k k} \\
0.37\end{array}$ & $\begin{array}{r}\mathbf{t r} \\
0.38\end{array}$ & $\begin{array}{c}\mathbf{j a *} \\
0.41\end{array}$ & Avg. \\
\hline \multicolumn{15}{|l|}{ Slots } \\
\hline base & 97.6 & 48.5 & 33.0 & 73.9 & 80.4 & 75.0 & 67.4 & 71.1 & 45.8 & 72.9 & 48.5 & 55.7 & 59.9 & 61.0 \\
\hline nmt-transfer & 0.0 & 50.9 & 34.5 & 60.8 & 63.7 & 51.0 & 41.3 & 54.2 & 48.2 & 27.9 & 0.2 & 52.0 & 45.0 & 44.1 \\
\hline aux-mlm & 97.3 & 53.0 & 34.6 & 75.9 & 82.2 & 78.0 & 63.8 & 69.5 & 48.1 & 69.4 & 51.3 & 58.4 & 63.5 & 62.3 \\
\hline aux-nmt & 0.0 & 44.5 & 33.3 & 71.4 & 76.9 & 71.9 & 58.5 & 62.9 & 38.7 & 70.3 & 38.2 & 50.2 & 58.7 & 56.3 \\
\hline aux-ud & 97.5 & 47.6 & 29.1 & 73.7 & 73.3 & 61.8 & 56.8 & 61.1 & 42.6 & 64.9 & 45.2 & 53.8 & 47.6 & 54.8 \\
\hline \multicolumn{15}{|l|}{ Intents } \\
\hline base & 99.7 & 67.8 & 74.2 & 87.5 & 72.3 & 81.7 & 75.7 & 80.7 & 63.1 & 83.3 & 60.1 & 74.7 & 53.9 & 72.9 \\
\hline nmt-transfer & 0.0 & 89.9 & 97.5 & 97.3 & 98.3 & 96.8 & 92.5 & 98.1 & 89.2 & 97.3 & 24.6 & 98.3 & 78.8 & 88.2 \\
\hline aux-mlm & 99.5 & 66.3 & 75.4 & 80.7 & 73.5 & 80.1 & 65.4 & 72.2 & 59.7 & 78.3 & 47.6 & 62.2 & 42.9 & 67.0 \\
\hline aux-nmt & 0.0 & 63.0 & 72.8 & 86.6 & 70.8 & 78.7 & 71.9 & 75.5 & 56.4 & 80.9 & 56.3 & 68.9 & 53.6 & 69.6 \\
\hline aux-ud & 99.5 & 62.6 & 58.2 & 67.7 & 60.1 & 62.9 & 59.9 & 65.0 & 45.7 & 70.4 & 24.6 & 43.9 & 38.5 & 55.0 \\
\hline XLM15 & en & de-st & de & da & $\mathrm{nl}$ & it & $\mathrm{sr}$ & id & ar & $\mathbf{z h}$ & $\mathrm{kk}$ & tr & $\mathrm{ja}^{*}$ & \\
\hline \multicolumn{15}{|l|}{ Slots } \\
\hline base & 97.0 & 39.4 & 33.3 & 26.3 & 30.9 & 27.3 & 15.9 & 14.9 & 49.1 & 57.6 & 10.9 & 45.5 & 33.4 & 32.1 \\
\hline nmt-transfer & 0.0 & 43.0 & 34.7 & 59.5 & 61.9 & 49.4 & 39.7 & 53.0 & 47.2 & 28.4 & 0.0 & 50.5 & 34.7 & 41.8 \\
\hline aux-mlm & 97.2 & 44.0 & 35.9 & 45.8 & 49.5 & 40.7 & 18.7 & 24.6 & 48.9 & 64.8 & 13.6 & 60.5 & 30.4 & 39.8 \\
\hline aux-nmt & 0.0 & 32.3 & 32.3 & 26.3 & 28.0 & 24.1 & 12.4 & 13.7 & 38.0 & 29.4 & 7.2 & 33.1 & 16.6 & 24.4 \\
\hline aux-ud & 97.0 & 46.0 & 34.6 & 36.3 & 45.4 & 45.3 & 22.0 & 21.6 & 45.1 & 52.5 & 13.1 & 50.1 & 33.2 & 37.1 \\
\hline \multicolumn{15}{|l|}{ Intents } \\
\hline base & 99.7 & 61.3 & 78.5 & 56.3 & 45.4 & 48.0 & 41.4 & 36.4 & 67.5 & 78.8 & 29.9 & 67.3 & 39.1 & 54.1 \\
\hline nmt-transfer & 0.0 & 80.6 & 97.6 & 97.6 & 97.7 & 96.6 & 92.4 & 96.7 & 88.4 & 97.1 & 16.7 & 97.9 & 61.7 & 85.1 \\
\hline aux-mlm & 99.6 & 63.9 & 86.3 & 62.8 & 59.9 & 53.0 & 31.4 & 42.1 & 64.0 & 86.5 & 25.7 & 63.3 & 44.4 & 56.9 \\
\hline aux-nmt & 0.0 & 52.0 & 52.0 & 60.7 & 44.7 & 44.7 & 40.3 & 43.1 & 54.3 & 54.1 & 21.0 & 53.2 & 25.2 & 45.4 \\
\hline aux-ud & 99.5 & 62.6 & 72.2 & 47.2 & 42.3 & 52.5 & 33.6 & 31.8 & 45.7 & 57.1 & 30.3 & 51.1 & 35.5 & 46.8 \\
\hline
\end{tabular}

Table 3: Results on slot labeling (in strict F1) and intent classification (in accuracy) on the development split of all 13 languages. Average over 5 seeds, standard deviations can be found in Appendix D. Sorted by language distance to en (de-st excluded), which is the cosine distance between the syntax, phonology and inventory vectors of lang2vec (Littell et al., 2017). Bold languages are included during pre-training. The last column (Avg.) is the average of all cross-lingual experiments, i.e. without English. *For Japanese we have 50\% less evaluation data.

auxiliary tasks, as opposed to previous hierarchical MTL work. For all tasks we use the default settings of MaChAmp and set the loss weight of each UD subtask to 0.25 .

Data For each language, we manually picked a matching UD treebank from version 2.6 (Nivre et al., 2020) (details in the Appendix). Whenever available, we picked an in-language treebank, otherwise we choose a related language. We used size, annotation quality, and domain as criteria.

\section{Results}

\subsection{Experimental Setup}

We target a low-resource setup, and hence all our experiments assume no target-language training nor development data for the target task. For all our experiments we use the English training from the Facebook and Snips data, and their English development sets (all converted to match our guidelines, see § 2). We use strict-span F1 score for slots (where both span and label must match exactly) and accuracy for intents as main evaluation metric as is standard for these tasks. ${ }^{11}$ All reported results (including analysis and test data) are the average over 5 runs with different random seeds.

To choose the final model, we use the scores on the English development data. We are aware that this was recently shown to be sub-optimal in some settings (Keung et al., 2020), however there is no clear solution on how to circumvent this in a pure zero-shot cross-lingual setup (i.e. without assuming any target language target task annotation data).

We use multilingual BERT (mBERT) as contextual encoder for our experiments. We are also interested in low-resource setups. As all of our languages are included in pre-training of mBERT (except the de-st dialect), we also study XLM15 (XLMMLM-TLM-XNLI15-1024), which in pre-training covers only 5 of the 13 XSID languages, to simulate further a real low-resource setup.

Table 3 reports the scores on 13 XSID languages, for 2 tasks (slot and intent prediction) and 2 pre-

\footnotetext{
${ }^{11}$ Ill-formed spans are automatically converted to match the BIO-scheme (first word with I is converted to B, and B-I spans with different labels are converted to all match the first label).
} 


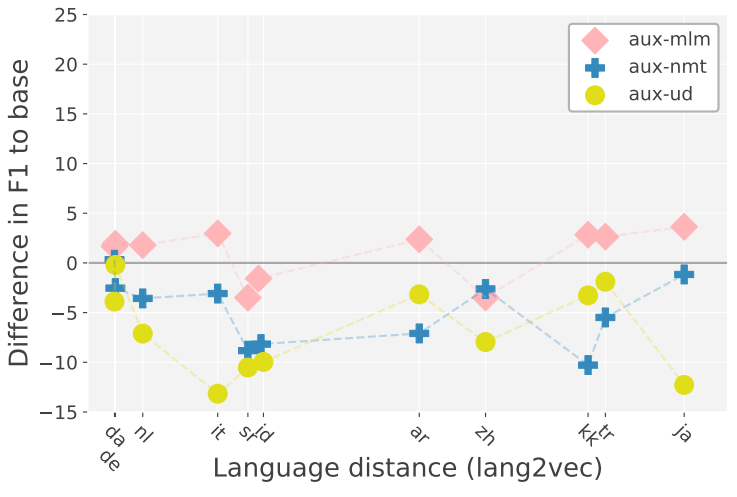

(a)

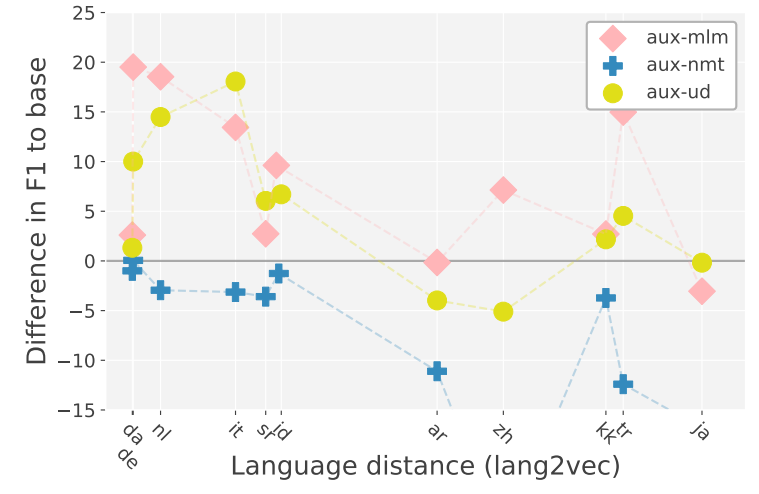

(b)

Figure 3: Performance increase over baseline for each auxiliary task with respect to the language distance (lang2vec) to English for mBERT (a) and XLM15 (b). It should be noted that the lines carry no meaning (i.e. we can not conclude performance based on language distance alone), and are shown to make trends visible.

trained language models. Languages are ordered by language distance, whenever available. Below we discuss the main findings per task.

Slots For slot filling, auxiliary tasks are beneficial for the majority of the languages, and the best performing multi-task model (aux-mlm) achieves +1.3 for mBERT and +7.7 for XLM15 average improvements over the baseline. By comparing mBERT and XLM15, there are significant performance drops for languages not seen during XLM15 pre-training, e.g., Danish (da) and Indonesian (id). This confirms that having a language in pre-training has a large impact on cross-lingual transfer for this task. For other languages involved in pre-training, both aux-mlm and aux-ud beat the baseline model. This supports our hypothesis that, after multilingual pre-training, auxiliary tasks (with token-level prediction both self-supervised and supervised) help the model learn the target language and a better latent alignment for cross-lingual slot filling.

Intents For intent classification the nmt-transfer model is very strong as it uses explicit translations, especially for languages not seen during pretraining. Using nmt as an auxiliary task does not come close, however, it should be noted that this only uses a fraction of the data and computational costs (see § 5.4). One main limitation of the nmttransfer model is that it is dependant on a highquality translation model, which in turn requires a large quantity of in-domain parallel data. Results on Kazakh (kk) confirm this, where the translation model is trained on out-of-domain data, because in-domain data was not available (§ 3.2).

\begin{tabular}{l|ll|ll}
\hline \multirow{2}{*}{ Model } & \multicolumn{2}{|c}{ XSID } & \multicolumn{2}{c}{ MultiAtis++ } \\
& Slots & Intents & Slots & Intents \\
\hline mBERT & & & & \\
\hline base & 61.00 & 72.91 & 71.12 & 87.28 \\
nmt-transfer & $44.13^{3}$ & $88.22^{11}$ & $49.20^{1}$ & $92.82^{8}$ \\
aux-mlm & $62.32^{9}$ & $67.02^{1}$ & $69.15^{1}$ & $83.33^{0}$ \\
aux-nmt & $56.29^{0}$ & $69.62^{0}$ & $66.95^{1}$ & $84.28^{0}$ \\
aux-ud & $54.81^{0}$ & $54.97^{0}$ & $54.18^{1}$ & $64.81^{0}$ \\
\hline XLM15 & & & & \\
\hline base & 32.05 & 54.15 & 22.71 & 70.63 \\
nmt-transfer & $41.85^{8}$ & $85.08^{11}$ & $20.97^{4}$ & $83.57^{8}$ \\
aux-mlm & $39.77^{10}$ & $56.94^{8}$ & $62.10^{8}$ & $81.54^{7}$ \\
aux-nmt & $23.85^{0}$ & $43.68^{2}$ & $0.31^{0}$ & $42.12^{0}$ \\
aux-ud & $37.10^{9}$ & $46.82^{1}$ & $52.95^{6}$ & $80.30^{7}$ \\
\hline
\end{tabular}

Table 4: Results on the test data, average over all languages except English. Significance tested with almost stochastic order (Dror et al., 2019) test with Bonferroni correction (Bonferroni, 1936) as implemented by U1mer (2021): 1,000 iterations $\mathrm{p}=0.05$. The number in superscript indicates the number of languages (/12) with significant improvements compared to the baseline.

\subsection{Test Data}

Our main findings are confirmed on the test data (Table 4), where we also evaluate on MultiAtis++. The nmt-transfer model perform superior on intents, whereas its performance on slots is worse. The best auxiliary setups are aux-mlm followed by aux-ud. Most significant gains with auxiliary tasks are obtained for languages not included in pre-training (XLM15). We believe there is a bug for aux-nmt with XLM15 (see also results in Appendix C), which we unfortunately could not resolve before submission time. Furthermore, we 


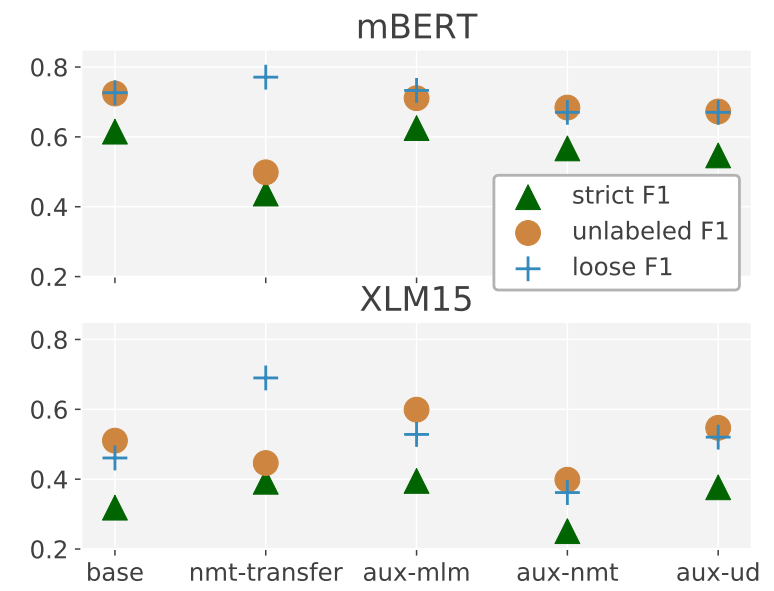

Figure 4: F1 scores variants for each model, averaged over 12 languages (English is not included).

believe more tuning of machine translation can increase its viability as auxiliary task. In general our results on MultiAtis++ are lower compared to $\mathrm{Xu}$ et al. (2020), which is probably because they used a black-box translation model.

\section{Analysis}

\subsection{Effect of Language Distance}

In Figure 3a we plot the performance increase over baseline for each auxiliary task with respect to the language distance when using mBERT. The results confirm that aux-mlm is the most promising auxiliary model, and clearly show that it is most beneficial for languages with a large distance to English Figure $3 b$ shows the same plot for the XLM15 models, and here the trends are quite different. First, we see that also for close languages, aux-ud as well as aux-mlm are beneficial. Second, the aux-ud model also performs better for the more distant languages.

\subsection{Slot Detection Versus Classification}

To evaluate whether the detection of the slots or the classification of the label is the bottleneck, we experiment with two varieties of the F1 score. For the first variant, we ignore the label and consider only whether the span is correct. We refer to this as unlabeled F1. For span detection, we allow for partial matches (but with the same label) which count towards true positives for precision and recall. We refer to this metric as loose F1.

Average scores with all three F1 scores for both pre-trained embeddings are plotted in Figure 4. One of the main findings is that nmt-transfer does very well on the loose F1 metric, which means that

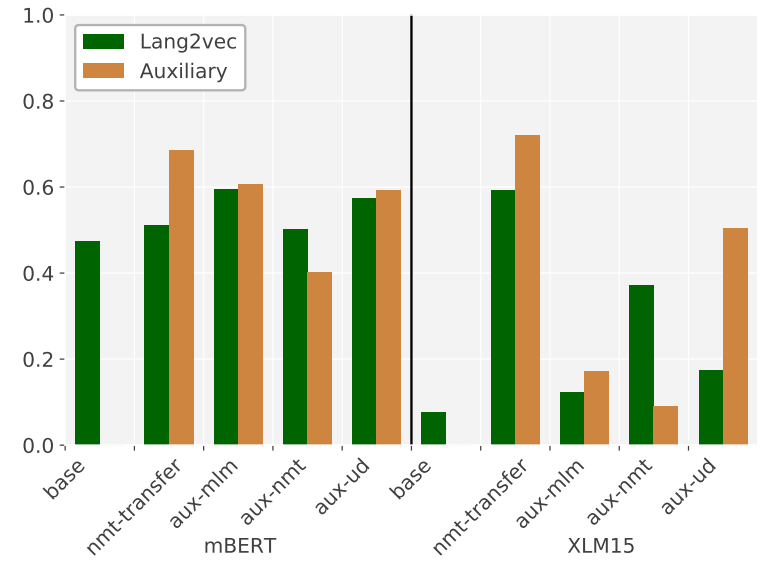

Figure 5: Pearson correlations between target tasks performance (average of slots/intents) and 1) language distance as estimated by lang2vec, and 2) the auxiliary task. For nmt-transfer, the auxiliary task is the BLEU score of the machine translation, and for the baseline there is no auxiliary task.

it is poor at finding spans, instead of labeling them. For the other models the difference between strict and unlabeled F1 is smaller, and both can gain approximately $5-10 \%$ absolute score for both types of errors. The only other large difference is for aux-nmt with XLM15, which makes more errors in the labeling (unlabeled F1 is higher). An analysis of the per-language results show that this is mainly due to errors made in the Kazakh dataset.

\subsection{Correlation Auxiliary Task Performance}

In Figure 5 we plot the absolute Pearson correlations between the auxiliary task (auxiliary task performance can be found in Appendix C) and the target tasks performance as well as between the target tasks and the language distance (from lang2vec, see Table 3). Here we use the average of slots/intents as score for the target task. The results show that when using only datasets from languages included in the pre-trained language model (i.e., mBERT), both language distance and auxiliary task performance are competitive predictors, whereas if also new languages are considered (XLM15) auxiliary task performance is clearly a stronger predictor.

\subsection{Computational Costs}

All experiments are executed on a single v100 Nvidia GPU. To compare computational costs, Table 5 reports the average training time over all languages for each of the models. The training time for nmt-transfer is the highest, followed by aux-nmt, then come the leaner auxiliary tasks. The inference 


\begin{tabular}{lr}
\hline Model & Time (minutes) \\
\hline base & 3 \\
nmt-transfer & 5,145 \\
aux-mlm & 220 \\
aux-nmt & 464 \\
aux-ud & 57 \\
\hline
\end{tabular}

Table 5: Average minutes to train a model, averaged over all languages and both embeddings. For nmttransfer we include the training of the NMT model.

time of all the models for the SLU tasks is highly similar due to the similar architecture (except for nmt-transfer requiring fairSeq a-priori).

\subsection{Case Study: Improving on de-st}

Our lowest-resource language variety de-st is not included in either embeddings, and the performance on it is generally low. To mitigate this, we investigate whether a small amount of raw data could improve the aux-mlm model. We scraped 23,572 tweets and 6,583 comments from ask.fm manually identified by a native speaker, and used these as auxiliary data in the aux-mlm model. Although this data is difficult to obtain and contains a mix including standard German and others, it resulted in an increase from 49.9 to 56.2 in slot F1 scores and from 68.0 to 68.7 for intents, compared to using the German data in aux-mlm, thereby largely outperforming the baseline. This shows that even small amounts of data are highly beneficial in aux training, confirming results of Muller et al. (2021).

\section{Related Work}

For related datasets, we refer to $\S 2.1$; in this section we will discuss different approaches on how to tackle cross-lingual SLU. Work on cross-lingual SLU can broadly be divided into two approaches, whether it is based mainly on parallel data or multilingual representations. The first stream of research focuses on generating training data in the target language with machine translation and mapping the slot labels through attention or an external word aligner. The translation-based approach can be further improved by filtering the resulting training data (Gaspers et al., 2018; Do and Gaspers, 2019), post-fixing the annotation by humans (Castellucci et al., 2019), or by using a soft-alignment based on attention, which alleviates error propagation and outperforms annotation projection using external word aligners (Xu et al., 2020).
The second stream of research uses multilingual representations. Upadhyay et al. (2018) use bilingual word embeddings based on Smith et al. (2017) in a bidirectional Long Short-Term Memory model for zero-shot SLU. Recent work focuses on finding better multilingual representations. Schuster et al. (2019) use a multilingual machine translation encoder as word representations. Liu et al. (2019b) propose refining the alignment of bilingual word representations. The best performing variants use contextualized BERT variants (Chen et al., 2019; Xu et al., 2020), which we depart from.

We propose a third, orthogonal line of research: joint target-language auxiliary task learning. We hypothesize that jointly training on target language auxiliary tasks helps to learn properties of the target language while learning a related task simultaneously. We frame masked language modeling, Universal Dependency parsing and machine translation as new auxiliary tasks for SLU.

Some work on SLU showed that syntax in graph convolution networks is beneficial for slots (Qin et al., 2020). Contemporary work shows that highresource English data helps target language modeling in sequential transfer setups (Phang et al., 2020). We focus on non-English target data for joint SLU in a single cross-lingual multi-task model instead.

\section{Conclusions}

We introduced XSID, a multilingual dataset for spoken language understanding with 13 languages from 6 language families, including an unstudied German dialect. XSID includes a wide variety of intent types and homogenized annotations. We propose non-English multi-task setups for zero-shot transfer to learn the target language: masked language modeling, neural machine translation and UD parsing. We compared the effect of these auxiliary tasks in two settings. Our results showed that masked language modeling led to the most stable performance improvements; however, when a language is not seen during pre-training, UD parsing led to an even larger performance increase. On the intents, generating target language training data using machine translation was outperforming all our proposed models, at a much higher computational cost however. Our analysis further shows that nmt-transfer struggles with span detection. Given training time and availability trade-off, MLM multitasking is a viable approach for SLU. 


\section{Acknowledgements}

We would like to thank Yiping Duan, Kristian Nørgaard Jensen, Illona Flecchi, Mike Zhang, and Caroline van der Goot for their annotation efforts. We thank Dennis Ulmer for his help with significance testing. Furthermore, we thank Fabian Triefenbach, Judith Gaspers and the anonymous reviewers for the feedback. We also thank NVIDIA, Google cloud computing and the ITU High-performance Computing cluster for computing resources. This research is supported in part by the Independent Research Fund Denmark (DFF) grant 9131-00019B and 9063-00077B and an Amazon Faculty Research (ARA) Award.

\section{References}

Dzmitry Bahdanau, Kyunghyun Cho, and Yoshua Bengio. 2015. Neural machine translation by jointly learning to align and translate. Proceedings of International Conference on Learning Representations (ICLR).

Loïc Barrault, Ondřej Bojar, Marta R. Costa-jussà, Christian Federmann, Mark Fishel, Yvette Graham, Barry Haddow, Matthias Huck, Philipp Koehn, Shervin Malmasi, Christof Monz, Mathias Müller, Santanu Pal, Matt Post, and Marcos Zampieri. 2019. Findings of the 2019 conference on machine translation (WMT19). In Proceedings of the Fourth Conference on Machine Translation (Volume 2: Shared Task Papers, Day 1), pages 1-61, Florence, Italy. Association for Computational Linguistics.

Valentina Bellomaria, Giuseppe Castellucci, Andrea Favalli, and Raniero Romagnoli. 2019. Almawaveslu: a new dataset for SLU in Italian. In Proceedings of the Sixth Italian Conference on Computational Linguistics.

Emily M. Bender and Batya Friedman. 2018. Data statements for natural language processing: Toward mitigating system bias and enabling better science. Transactions of the Association for Computational Linguistics, 6:587-604.

Carlo Bonferroni. 1936. Teoria statistica delle classi e calcolo delle probabilita. Pubblicazioni del R Istituto Superiore di Scienze Economiche e Commericiali di Firenze, 8:3-62.

Giuseppe Castellucci, Valentina Bellomaria, Andrea Favalli, and Raniero Romagnoli. 2019. Multilingual intent detection and slot filling in a joint BERT-based model. arXiv:1907.02884 [cs]. ArXiv: 1907.02884.

Mauro Cettolo, Niehues Jan, Stüker Sebastian, Luisa Bentivogli, Roldano Cattoni, and Marcello Federico. 2016. The IWSLT 2016 evaluation campaign. In
International Workshop on Spoken Language Translation.

Qian Chen, Zhu Zhuo, and Wen Wang. 2019. Bert for joint intent classification and slot filling. arXiv preprint: 1902.10909 .

Kyunghyun Cho, Bart van Merriënboer, Caglar Gulcehre, Dzmitry Bahdanau, Fethi Bougares, Holger Schwenk, and Yoshua Bengio. 2014. Learning phrase representations using RNN encoder-decoder for statistical machine translation. In Proceedings of the 2014 Conference on Empirical Methods in Natural Language Processing (EMNLP), pages 17241734, Doha, Qatar. Association for Computational Linguistics.

Stephane Clinchant, Kweon Woo Jung, and Vassilina Nikoulina. 2019. On the use of BERT for neural machine translation. In Proceedings of the 3rd Workshop on Neural Generation and Translation, pages 108-117, Hong Kong. Association for Computational Linguistics.

Alexis Conneau, Kartikay Khandelwal, Naman Goyal, Vishrav Chaudhary, Guillaume Wenzek, Francisco Guzmán, Edouard Grave, Myle Ott, Luke Zettlemoyer, and Veselin Stoyanov. 2020. Unsupervised cross-lingual representation learning at scale. In Proceedings of the 58th Annual Meeting of the Association for Computational Linguistics, pages 84408451, Online. Association for Computational Linguistics.

Alexis Conneau and Guillaume Lample. 2019. Crosslingual language model pretraining. In Advances in Neural Information Processing Systems, pages 7059-7069.

Alice Coucke, Alaa Saade, Adrien Ball, Théodore Bluche, Alexandre Caulier, David Leroy, Clément Doumouro, Thibault Gisselbrecht, Francesco Caltagirone, Thibaut Lavril, et al. 2018. Snips voice platform: an embedded spoken language understanding system for private-by-design voice interfaces. arXiv preprint: 1805.10190.

Jacob Devlin, Ming-Wei Chang, Kenton Lee, and Kristina Toutanova. 2019. BERT: Pre-training of deep bidirectional transformers for language understanding. In Proceedings of the 2019 Conference of the North American Chapter of the Association for Computational Linguistics: Human Language Technologies, Volume 1 (Long and Short Papers), pages 4171-4186, Minneapolis, Minnesota. Association for Computational Linguistics.

Quynh Do and Judith Gaspers. 2019. Cross-lingual transfer learning with data selection for large-scale spoken language understanding. In Proceedings of the 2019 Conference on Empirical Methods in Natural Language Processing and the 9th International Joint Conference on Natural Language Processing (EMNLP-IJCNLP), pages 1455-1460, Hong Kong, China. Association for Computational Linguistics. 
Rotem Dror, Segev Shlomov, and Roi Reichart. 2019 Deep dominance - how to properly compare deep neural models. In Proceedings of the 57th Conference of the Association for Computational Linguistics, ACL 2019, Florence, Italy, July 28-August 2, 2019, Volume 1: Long Papers, pages 2773-2785. Association for Computational Linguistics.

Arash Einolghozati, Abhinav Arora, Lorena SainzMaza Lecanda, Anuj Kumar, and Sonal Gupta. 2021. El volumen louder por favor: Code-switching in task-oriented semantic parsing. In Proceedings of the 16th Conference of the European Chapter of the Association for Computational Linguistics: Main Volume, pages 1009-1021, Online. Association for Computational Linguistics.

Joseph L Fleiss. 1971. Measuring nominal scale agreement among many raters. Psychological bulletin, 76(5):378.

Matt Gardner, Joel Grus, Mark Neumann, Oyvind Tafjord, Pradeep Dasigi, Nelson F. Liu, Matthew Peters, Michael Schmitz, and Luke Zettlemoyer. 2018. AllenNLP: A deep semantic natural language processing platform. In Proceedings of Workshop for NLP Open Source Software (NLP-OSS), pages 16, Melbourne, Australia. Association for Computational Linguistics.

Judith Gaspers, Penny Karanasou, and Rajen Chatterjee. 2018. Selecting machine-translated data for quick bootstrapping of a natural language understanding system. In Proceedings of the 2018 Conference of the North American Chapter of the Association for Computational Linguistics: Human Language Technologies, Volume 3 (Industry Papers), pages 137-144, New Orleans - Louisiana. Association for Computational Linguistics.

Jonathan Godwin, Pontus Stenetorp, and Sebastian Riedel. 2016. Deep semi-supervised learning with linguistically motivated sequence labeling task hierarchies. arXiv preprint: 1612.09113.

Ian J Goodfellow, Mehdi Mirza, Da Xiao, Aaron Courville, and Yoshua Bengio. 2014. An empirical investigation of catastrophic forgetting in gradient-based neural networks. Proceedings of International Conference on Learning Representations (ICLR).

Suchin Gururangan, Ana Marasović, Swabha Swayamdipta, Kyle Lo, Iz Beltagy, Doug Downey, and Noah A. Smith. 2020. Don't stop pretraining: Adapt language models to domains and tasks. In Proceedings of the 58th Annual Meeting of the Association for Computational Linguistics, pages 8342-8360, Online. Association for Computational Linguistics.

Harald Hammarström and Sebastian Nordhoff. 2011. Langdoc: Bibliographic infrastructure for linguistic typology. Oslo Studies in Language, 3(2):31-43.
Kazuma Hashimoto, Caiming Xiong, Yoshimasa Tsuruoka, and Richard Socher. 2017. A joint many-task model: Growing a neural network for multiple NLP tasks. In Proceedings of the 2017 Conference on Empirical Methods in Natural Language Processing, pages 1923-1933, Copenhagen, Denmark. Association for Computational Linguistics.

Xiaodong He, Li Deng, Dilek Hakkani-Tur, and Gokhan Tur. 2013. Multi-style adaptive training for robust cross-lingual spoken language understanding. In 2013 IEEE International Conference on Acoustics, Speech and Signal Processing, pages 83428346. IEEE.

Charles T. Hemphill, John J. Godfrey, and George R. Doddington. 1990. The ATIS spoken language systems pilot corpus. In Speech and Natural Language: Proceedings of a Workshop Held at Hidden Valley, Pennsylvania, June 24-27,1990.

Kenji Imamura and Eiichiro Sumita. 2019. Recycling a pre-trained BERT encoder for neural machine translation. In Proceedings of the 3rd Workshop on Neural Generation and Translation, pages 23-31, Hong Kong. Association for Computational Linguistics.

Phillip Keung, Yichao Lu, Julian Salazar, and Vikas Bhardwaj. 2020. Don't use English dev: On the zero-shot cross-lingual evaluation of contextual embeddings. In Proceedings of the 2020 Conference on Empirical Methods in Natural Language Processing (EMNLP), pages 549-554, Online. Association for Computational Linguistics.

Pierre Lison and Jörg Tiedemann. 2016. OpenSubtitles2016: Extracting large parallel corpora from movie and TV subtitles. In Proceedings of the Tenth International Conference on Language Resources and Evaluation (LREC'16), pages 923-929, Portorož, Slovenia. European Language Resources Association (ELRA).

Patrick Littell, David R. Mortensen, Ke Lin, Katherine Kairis, Carlisle Turner, and Lori Levin. 2017. URIEL and lang2vec: Representing languages as typological, geographical, and phylogenetic vectors. In Proceedings of the 15th Conference of the European Chapter of the Association for Computational Linguistics: Volume 2, Short Papers, pages 8-14, Valencia, Spain. Association for Computational Linguistics.

Yinhan Liu, Myle Ott, Naman Goyal, Jingfei Du, Mandar Joshi, Danqi Chen, Omer Levy, Mike Lewis, Luke Zettlemoyer, and Veselin Stoyanov. 2019a. RoBERTa: A robustly optimized bert pretraining approach. arXiv preprint: 1907.11692.

Zihan Liu, Jamin Shin, Yan Xu, Genta Indra Winata, Peng Xu, Andrea Madotto, and Pascale Fung. 2019b. Zero-shot cross-lingual dialogue systems with transferable latent variables. In Proceedings of the 2019 Conference on Empirical Methods in Natural Language Processing and the 9th International 
Joint Conference on Natural Language Processing (EMNLP-IJCNLP), pages 1297-1303, Hong Kong, China. Association for Computational Linguistics.

Michael McCloskey and Neal J Cohen. 1989. Catastrophic interference in connectionist networks: The sequential learning problem. In Psychology of learning and motivation, volume 24, pages 109-165. Elsevier.

Benjamin Muller, Antonis Anastasopoulos, Benoît Sagot, and Djamé Seddah. 2021. When being unseen from mbert is just the beginning: Handling new languages with multilingual language models. In Proceedings of the 2021 Conference of the North American Chapter of the Association for Computational Linguistics: Human Language Technologies, Volume 1 (Long and Short Papers). Association for Computational Linguistics.

Joakim Nivre, Marie-Catherine de Marneffe, Filip Ginter, Jan Hajič, Christopher D. Manning, Sampo Pyysalo, Sebastian Schuster, Francis Tyers, and Daniel Zeman. 2020. Universal Dependencies v2: An evergrowing multilingual treebank collection. In Proceedings of the 12th Language Resources and Evaluation Conference, pages 4034-4043, Marseille, France. European Language Resources Association.

Myle Ott, Sergey Edunov, Alexei Baevski, Angela Fan, Sam Gross, Nathan Ng, David Grangier, and Michael Auli. 2019. fairseq: A fast, extensible toolkit for sequence modeling. In Proceedings of the 2019 Conference of the North American Chapter of the Association for Computational Linguistics (Demonstrations), pages 48-53, Minneapolis, Minnesota. Association for Computational Linguistics.

Jason Phang, Iacer Calixto, Phu Mon Htut, Yada Pruksachatkun, Haokun Liu, Clara Vania, Katharina Kann, and Samuel R. Bowman. 2020. English intermediate-task training improves zero-shot crosslingual transfer too. In Proceedings of the 1st Conference of the Asia-Pacific Chapter of the Association for Computational Linguistics and the 10th International Joint Conference on Natural Language Processing, pages 557-575, Suzhou, China. Association for Computational Linguistics.

Libo Qin, Minheng Ni, Yue Zhang, Wanxiang Che, Yangming Li, and Ting Liu. 2020. Multi-domain spoken language understanding using domain- and task-aware parameterization. arXiv:2004.14871 [cs]. ArXiv: 2004.14871.

Sebastian Schuster, Sonal Gupta, Rushin Shah, and Mike Lewis. 2019. Cross-lingual transfer learning for multilingual task oriented dialog. In Proceedings of the 2019 Conference of the North American Chapter of the Association for Computational Linguistics: Human Language Technologies, Volume 1 (Long and Short Papers), pages 3795-3805, Minneapolis, Minnesota. Association for Computational Linguistics.
Rico Sennrich, Barry Haddow, and Alexandra Birch. 2016. Neural machine translation of rare words with subword units. In Proceedings of the 54th Annual Meeting of the Association for Computational Linguistics (Volume 1: Long Papers), pages 17151725, Berlin, Germany. Association for Computational Linguistics.

Samuel L Smith, David HP Turban, Steven Hamblin, and Nils Y Hammerla. 2017. Offline bilingual word vectors, orthogonal transformations and the inverted softmax. In Proceedings of International Conference on Learning Representations (ICLR).

Marcin Sowański and Artur Janicki. 2020. Leyzer: A dataset for multilingual virtual assistants. In International Conference on Text, Speech, and Dialogue, pages 477-486. Springer.

Pontus Stenetorp, Sampo Pyysalo, Goran Topić, Tomoko Ohta, Sophia Ananiadou, and Jun'ichi Tsujii. 2012. brat: a web-based tool for NLP-assisted text annotation. In Proceedings of the Demonstrations at the 13th Conference of the European Chapter of the Association for Computational Linguistics, pages 102-107, Avignon, France. Association for Computational Linguistics.

Ilya Sutskever, Oriol Vinyals, and Quoc V Le. 2014. Sequence to sequence learning with neural networks. In Advances in neural information processing systems, pages 3104-3112.

Jörg Tiedemann. 2012. Parallel data, tools and interfaces in OPUS. In Proceedings of the Eighth International Conference on Language Resources and Evaluation (LREC'12), pages 2214-2218, Istanbul, Turkey. European Language Resources Association (ELRA).

Dennis Ulmer. 2021. deep-significance: Easy and better significance testing for deep neural networks. Https:/github.com/Kaleidophon/deep-significance.

Shyam Upadhyay, Manaal Faruqui, Gokhan Tür, Hakkani-Tür Dilek, and Larry Heck. 2018. (Almost) zero-shot cross-lingual spoken language understanding. In 2018 IEEE International Conference on Acoustics, Speech and Signal Processing (ICASSP), pages 6034-6038. IEEE.

Rob van der Goot, Ahmet Üstün, Alan Ramponi, Ibrahim Sharaf, and Barbara Plank. 2021. Massive choice, ample tasks (MaChAmp): A toolkit for multi-task learning in NLP. In Proceedings of the 16th Conference of the European Chapter of the Association for Computational Linguistics: System Demonstrations, pages 176-197, Online. Association for Computational Linguistics.

Ashish Vaswani, Noam Shazeer, Niki Parmar, Jakob Uszkoreit, Llion Jones, Aidan N Gomez, Łukasz Kaiser, and Illia Polosukhin. 2017. Attention is all you need. In Advances in neural information processing systems, pages 5998-6008. 
Pawel Swietojanski Xingkun Liu, Arash Eshghi and Verena Rieser. 2019. Benchmarking natural language understanding services for building conversational agents. In Proceedings of the Tenth International Workshop on Spoken Dialogue Systems Technology (IWSDS), Ortigia, Siracusa (SR), Italy. Springer.

Weijia Xu, Batool Haider, and Saab Mansour. 2020. End-to-end slot alignment and recognition for crosslingual NLU. In Proceedings of the 2020 Conference on Empirical Methods in Natural Language Processing (EMNLP), pages 5052-5063, Online. Association for Computational Linguistics. 
Appendix

\begin{tabular}{lr}
\hline Parameter & Value \\
\hline Optimizer & Adam \\
$\beta_{1}, \beta_{2}$ & $0.9,0.99$ \\
Dropout & 0.3 \\
Epochs & 20 \\
Batch size & 32 \\
Learning rate & 0.001 \\
Weight decay & 0.01 \\
LR scheduler & slanted triangular \\
Decay factor & 0.38 \\
Cut frac & 0.2 \\
\hline
\end{tabular}

Table 6: Hyperparameter setting used in the experiments

\section{A Hyperparameter settings}

Hyperparameters follow the default setting of MaChAmp 0.2 (van der Goot et al., 2021) with 20 epochs, as reported in Table 6.

\section{B Auxiliary Datasets}

Table 7 reports the data sources for the treebanks and the dataset sizes in number of words and sentences for both the treebanks and the parallel data.

\section{Scores on auxiliary tasks}

Even though it was not our goal to improve the auxiliary tasks, performance on these can still be relevant to analyze whether there is any correlation to performance on the XSID tasks. In Table 8, we report the full results for all tasks. These are the scores the correlations of Figure 5 are based on.

\section{Standard Deviations}

Standard deviations of our main results (Table 3) are shown in Table 9.

\section{E XSID Data Statement}

Following (Bender and Friedman, 2018), the following outlines the data statement for XSID:

A. CURATION RATIONALE Collection of utterances intended to be used for digital assistants, generated by crowd-workers. We selected a random sample from two much larger sets (Coucke et al., 2018; Schuster et al., 2019) which we translated and annotated for slots and intents for the cross-lingual study of SLU.
B. LANGUAGE VARIETY The English data was created by native English speakers and all translations are translated by native speakers. We translated to the following languages according to the iso 639-3 codes: 'deu', 'jpn', 'tur', 'nld', 'ita', 'dan', 'arb', 'kaz', 'srp', 'eng', 'ind', 'cmn'. Southtyrolean does not have an iso 693-3 language code.

C. SPEAKER DEMOGRAPHIC The original data is generated by crowd-workers and their demographics are unknown.

D. ANNOTATOR DEMOGRAPHIC Translators and annotators are the same people. Their age ranges from 20 to 57 , with the majority being below 30, almost all annotators have a background in NLP (except for Chinese, and one inter-annotator for Dutch). Most annotators are currently doing a $\mathrm{PhD}$, whereas there is one postdoc and two faculty.

E. SPEECH SITUATION The original data is generated in June 2017 (Coucke et al., 2018) and probably in 2019 (Schuster et al., 2019). The crowd workers were tasked to type sentences as how they would ask them in spoken form to a digitial assistant given a topic (intent).

F. TEXT CHARACTERISTICS The genre of the data is determined by the set of supported intents:

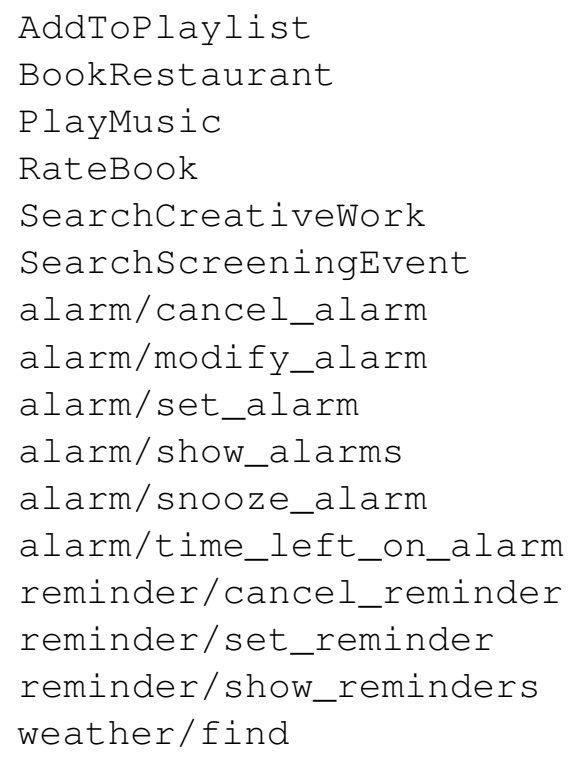

I. PROVENANCE APPENDIX The original datasets have been released with the following licenses:

- Schuster et al. (2019): CC-BY-SA license.

- Coucke et al. (2018): CC0 1.0 Universal

We use the CC-BY-SA license for our redistribution of the data. 


\begin{tabular}{l|lrr|rr}
\hline & \multicolumn{3}{|c}{ Universal Dependencies } & \multicolumn{2}{c}{ Parallel data } \\
Lang. & Treebank & \#sents & \#words & \#sents & \#words \\
\hline ar & UD_Arabic-PADT & 6,075 & 191,869 & $22,666,885$ & $122,580,047$ \\
da & UD_Danish-DDT & 4,383 & 80,378 & $11,021,827$ & $71,415,893$ \\
de & UD_German-GSD & 13,814 & 259,194 & $14,325,270$ & $99,354,451$ \\
en & UD_English-EWT & 12,543 & 204,585 & 0 & 0 \\
es & UD_Spanish-GSD & 14,187 & 375,149 & $61,434,251$ & $415,369,072$ \\
fr & UD_French-GSD & 14,449 & 344,970 & $41,921,465$ & $280,924,433$ \\
hi & UD_Hindi-HDTB & 13,304 & 281,057 & 93,016 & 620,929 \\
id & UD_Indonesian-GSD & 4,477 & 97,531 & $5,370,460$ & $30,758,822$ \\
it & UD_Italian-ISDT & 13,121 & 257,616 & $26,344,624$ & $180,169,211$ \\
ja & UD_Japanese-GSD & 7,027 & 167,482 & $1,883,365$ & $12,891,698$ \\
kk & UD_Kazakh-KTB & 1,047 & 9,872 & 595,060 & $10,058,764$ \\
nl & UD_Dutch-LassySmall & 5,787 & 75,080 & $28,835,007$ & $196,968,670$ \\
pt & UD_Portuguese-GSD & 9,664 & 238,714 & $33,375,963$ & $218,626,646$ \\
sr & UD_Serbian-SET & 3,328 & 74,259 & $22,319,620$ & $133,297,245$ \\
th & UD_Thai-PUD & 1,000 & 22,322 & $3,281,533$ & $4,332,396$ \\
tr & UD_Turkish-IMST & 3,664 & 36,822 & $45,788,547$ & $229,132,015$ \\
zh & UD_Chinese-GSD & 3,997 & 98,616 & $9,475,118$ & $89,458,907$ \\
\hline
\end{tabular}

Table 7: Dataset sizes for auxiliary tasks, for our auxiliary setting we constrained the model to only use 10,000 sentences of the treebanks and 100,000 of the parallel data

\begin{tabular}{l|rrrr|rrrr}
\hline Lang & ud(avg.) & mlm & aux-nmt & nmt-transfer & ud(avg.) & mlm & aux-nmt & nmt-transfer \\
\hline & mBERT & & & & XLM15 & & & \\
\hline ar & 88.12 & 1.81 & 11.97 & 16.78 & 88.51 & 3.21 & 1.01 & 16.78 \\
da & 94.10 & 3.17 & 13.86 & 56.24 & 89.35 & 3.00 & 9.80 & 56.24 \\
de & 93.00 & 1.87 & 14.22 & 25.93 & 92.83 & 2.63 & 1.19 & 25.93 \\
en & 95.24 & 4.17 & 0.00 & 0.00 & 94.90 & 5.18 & 0.00 & 0.00 \\
id & 91.31 & 2.32 & 20.89 & 27.96 & 88.02 & 1.13 & 4.02 & 27.96 \\
it & 96.83 & 4.03 & 8.98 & 44.73 & 95.46 & 2.52 & 7.17 & 44.73 \\
ja & 97.97 & 2.91 & 4.53 & 10.08 & 97.12 & 2.79 & 1.53 & 10.08 \\
kk & 70.55 & 1.48 & 2.67 & 0.00 & 52.08 & 2.65 & 0.09 & 0.00 \\
nl & 93.84 & 3.81 & 11.66 & 53.43 & 90.14 & 2.59 & 9.50 & 53.43 \\
sr & 94.18 & 3.54 & 9.78 & 35.50 & 89.81 & 4.14 & 7.25 & 35.50 \\
tr & 83.04 & 1.71 & 9.31 & 14.45 & 79.41 & 2.87 & 0.87 & 14.45 \\
zh & 94.80 & 1.52 & 11.52 & 20.31 & 93.00 & 2.01 & 0.12 & 20.31 \\
\hline
\end{tabular}

Table 8: Results on auxiliary tasks: for UD, we use the average over UPOS accuracy, lemma accuracy, morphological feature accuracy (all features as 1 label) and depdendency LAS, for masked language modeling (mlm) we use perplexity, and the last two columns (nmt) are bleu scores. 


\begin{tabular}{|c|c|c|c|c|c|c|c|c|c|c|c|c|c|c|}
\hline $\begin{array}{l}\text { mBERT } \\
\text { lang2vec }\end{array}$ & en & $\frac{\text { de-st }}{-}$ & $\begin{array}{r}\text { de } \\
0.18\end{array}$ & $\begin{array}{r}\text { da } \\
0.18\end{array}$ & $\begin{array}{r}\mathbf{n l} \\
0.19\end{array}$ & $\begin{array}{r}\text { it } \\
0.22\end{array}$ & $\begin{array}{r}\mathbf{s r} \\
0.23\end{array}$ & $\begin{array}{r}\text { id } \\
0.24\end{array}$ & $\begin{array}{r}\text { ar } \\
0.30\end{array}$ & $\begin{array}{r}\mathbf{z h} \\
0.33\end{array}$ & $\begin{array}{r}\mathbf{k k} \\
0.37\end{array}$ & $\begin{array}{r}\mathbf{t r} \\
0.38\end{array}$ & $\begin{array}{c}\mathbf{j a}^{*} \\
0.41\end{array}$ & Avg. \\
\hline \multicolumn{15}{|l|}{ Slots } \\
\hline base & 0.2 & 4.8 & 3.1 & 1.7 & 1.0 & 1.0 & 2.4 & 2.2 & 1.3 & 2.3 & 4.1 & 1.4 & 6.5 & 2.6 \\
\hline nmt-transfer & 0.0 & 2.0 & 0.7 & 1.3 & 1.7 & 1.4 & 1.6 & 1.4 & 1.2 & 1.1 & 0.3 & 1.7 & 2.8 & 1.4 \\
\hline aux-mlm & 0.4 & 1.8 & 0.9 & 1.9 & 1.3 & 0.9 & 2.0 & 2.3 & 3.0 & 4.0 & 2.0 & 3.1 & 3.1 & 2.2 \\
\hline aux-nmt & 0.0 & 1.9 & 1.3 & 1.3 & 1.1 & 1.3 & 3.2 & 1.9 & 2.1 & 2.3 & 1.9 & 2.6 & 4.9 & 2.1 \\
\hline aux-ud & 0.2 & 1.4 & 1.0 & 0.7 & 1.7 & 4.0 & 1.1 & 2.9 & 0.3 & 1.8 & 1.4 & 2.8 & 3.5 & 1.9 \\
\hline \multicolumn{15}{|l|}{ Intents } \\
\hline base & 0.0 & 4.5 & 3.5 & 2.8 & 2.0 & 2.0 & 2.5 & 3.4 & 2.4 & 1.8 & 3.1 & 1.1 & 3.8 & 2.8 \\
\hline nmt-transfer & 0.0 & 2.9 & 0.6 & 1.0 & 0.5 & 1.1 & 2.4 & 0.6 & 0.5 & 0.8 & 6.6 & 0.4 & 4.2 & 1.8 \\
\hline aux-mlm & 0.2 & 2.6 & 2.6 & 2.0 & 2.6 & 4.2 & 4.6 & 7.2 & 3.4 & 2.3 & 4.5 & 5.9 & 8.9 & 4.2 \\
\hline aux-nmt & 0.0 & 4.2 & 2.7 & 2.0 & 3.0 & 4.2 & 2.7 & 1.6 & 2.5 & 3.6 & 3.3 & 3.6 & 5.7 & 3.3 \\
\hline aux-ud & 0.2 & 3.2 & 3.2 & 4.0 & 1.8 & 3.9 & 4.4 & 3.8 & 5.2 & 3.5 & 1.2 & 3.5 & 4.1 & 3.5 \\
\hline XLM15 & en & de-st & de & da & $\mathrm{nl}$ & it & $\mathrm{sr}$ & id & ar & $\mathbf{z h}$ & $\mathrm{kk}$ & tr & $\mathrm{ja}^{*}$ & \\
\hline \multicolumn{15}{|l|}{ Slots } \\
\hline base & 0.3 & 2.3 & 1.5 & 1.7 & 1.7 & 2.0 & 3.7 & 0.9 & 1.2 & 4.0 & 1.1 & 2.9 & 3.4 & 2.2 \\
\hline nmt-transfer & 0.0 & 4.3 & 1.0 & 2.0 & 0.9 & 1.0 & 1.6 & 1.8 & 1.1 & 2.7 & 0.0 & 1.5 & 19.5 & 3.1 \\
\hline aux-mlm & 0.4 & 3.1 & 0.4 & 4.0 & 3.6 & 1.4 & 1.2 & 3.1 & 1.4 & 2.7 & 1.3 & 2.0 & 8.5 & 2.7 \\
\hline aux-nmt & 0.0 & 4.1 & 4.1 & 2.2 & 2.0 & 1.6 & 2.4 & 0.8 & 3.0 & 17.5 & 3.9 & 1.9 & 5.2 & 4.1 \\
\hline aux-ud & 0.3 & 2.4 & 0.5 & 3.5 & 3.7 & 1.8 & 3.9 & 4.1 & 1.6 & 1.8 & 3.0 & 2.2 & 4.9 & 2.8 \\
\hline \multicolumn{15}{|l|}{ Intents } \\
\hline base & 0.2 & 5.3 & 2.0 & 6.1 & 6.0 & 6.7 & 4.5 & 2.8 & 3.1 & 5.4 & 10.0 & 3.2 & 7.0 & 5.2 \\
\hline nmt-transfer & 0.0 & 3.9 & 1.2 & 0.7 & 1.0 & 0.8 & 2.4 & 1.0 & 0.4 & 1.0 & 6.4 & 0.5 & 8.3 & 2.3 \\
\hline aux-mlm & 0.1 & 2.1 & 2.0 & 4.6 & 6.0 & 2.6 & 7.9 & 8.3 & 4.2 & 1.3 & 6.0 & 7.0 & 4.6 & 4.7 \\
\hline aux-nmt & 0.0 & 5.1 & 5.1 & 2.0 & 1.6 & 3.1 & 4.8 & 3.0 & 5.3 & 13.9 & 10.1 & 3.7 & 11.5 & 5.8 \\
\hline aux-ud & 0.3 & 1.6 & 2.7 & 3.9 & 4.0 & 4.6 & 4.4 & 5.0 & 4.4 & 4.1 & 4.6 & 5.8 & 3.2 & 4.0 \\
\hline
\end{tabular}

Table 9: Standard deviation matching all results from our main results (Table 3)

\section{F Translation Guidelines}

We aim to provide a fluent translation which is as similar (in meaning) as possible to the original. In some cases translations naturally have more distance, i.e. ' $7 \mathrm{pm}$ ' might translate to ' 7 in the evening' for languages in which there is no equivalent for 'pm'. The goal is to obtain sentences as they could possibly be used in the target language. Some general guidelines:

- In general, named entities are not translated, with the exception of place names, like cities and countries. So names of playlists, persons etc. stay the same, and things mentioned between quotes as well. In languages where names are often transcribed differently (i.e. Serbian), this is done during annotation.

- In case of grammatical mistakes, they are kept (if possible) in the target translation.

- We keep capitalization and punctuation as in the original data (if they exist in the target language).

- Abbreviations not common in fluent discourse are expanded (e.g., Wed $\mapsto$ "mercoledi", meds $\mapsto$ medicin), also words that do not exist in the target language are paraphrased: 'whats the high tomorrow' $\mapsto$ 'whats the maximum temperature tomorrow'.

- Some things can not be translated directly. For example, the phrase 'play me $\mathrm{X}$ ' does not exist in many languages. E.g. 'me' might not be translated.

- Possessive determiners (e.g. "my") should be preserved and translated whenever possible.

- For languages in which words are not separated by whitespace (i.e. Japanese and Chinese), we ask the translator to include whitespaces at word boundaries to simplify the annotation of the slots. 


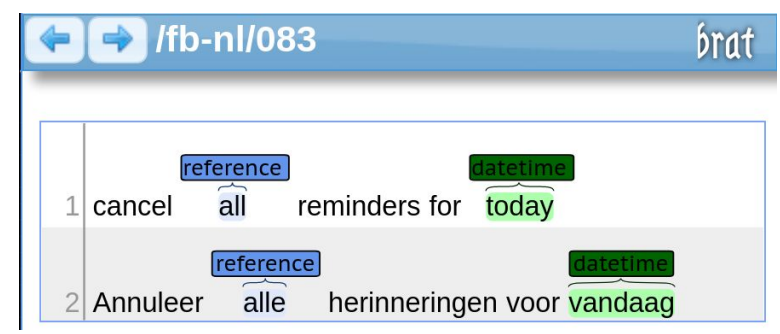

Figure 6: Example showing the annotation of a Dutch instance in Brat

\section{G Annotation Guidelines}

This section describes our annotation guidelines. The aim of these guidelines was to make annotations homogeneous across earlier efforts for which guidelines were not available. Two major changes compared to the original annotations include: i) to generalize labels to make them more broadly applicable to other intent subtypes (an example is the recurrent datetime event from the Facebook data (Schuster et al., 2019), which was originally only applied to reminders and not to alarms, as discussed below); ii) we drop annotations of nouns as slots which are directly inferrable from the intent label (e.g. the 'reminder/noun' label was only applicable to nouns, but it was sometimes expressed as a verb and hence annotations were missing; as they are already annotated in the sentence-level intent slots, we drop such obvious slots).

For the annotation, we use Brat (Stenetorp et al., 2012), and provided the annotators with the gold English annotation (see Figure 6). English annotation was conducted by three annotators who discussed and resolved any initial disagreements. For annotation of the other languages, annotators were instructed to follow the English annotation when possible to maintain consistency.

Because no annotation guidelines were released with the original data, we provide guidelines for our re-annotation of the slots below. Examples are shown in Figure 7.

Spans We exclude function words in the beginning of an NP or VP, like 'for', 'from' in the examples above. An exception is when it is in the middle of a span as contiguous slots are preferred, like in example 2. This is different from previous releases of the data (Schuster et al., 2019), where datetime included 'for', 'at', 'to' and 'on'. We decided to drop them to make the annotations more homogeneous across slot labels, while capturing the core ('head spans') of the slots.
When two words of the same type occur sequentially, we annotate them as one span. This happens both for datetime (example 2, [5 to $6 \mathrm{am}$ ]) as well as reference (example 6, [all my]). Furthermore, we keep the annotation on the word-level to simplify processing. If only a part of a word belongs to a label, we annotate the whole word with that label.

Slot labels After our adaptations of the original labels, we annotate the following labels:

- datetime: Indicating a date or a time. Only concrete times are annotated (not, 'until deleted', 'what time' or 'when'), and times relative to other events are included (e.g. 'after work', 'later'). Non-concrete times, like 'until deleted' (example 9) are excluded.

- recurring_datetime: a recurring event, can be used for alarms and reminders. This category prioritizes over datetime. Example: 'make alarm for [weekdays at $7 \mathrm{am}$ ]', if at least one recurring_datetime exists in an instance, all datetimes should be annotated as recurring_datetime (even if they are in different spans, see example 9).

- location: describes a location; can be a proper noun (like 'New York') or a nominal or adjective referring to a location ('my

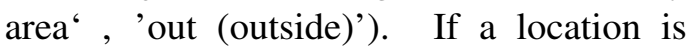
part of a reminder item, it is annotated as reminder/todo instead.

- reference: modifies the scope of an alarm or reminder, usually 'my' or 'all' used in front of the word 'reminder(s)' or 'alarm(s)'. Multiple sequential references are annotated as one span ('cancel [all my] alarms').

- reminder/todo: the item that should be reminded, the word 'to' should be excluded. In special cases, we also apply this for alarms (see example 8).

- weather/attribute: A property that describes an aspect of the weather; e.g. 'cold', 'rain', 'temperature', 'severe'. Also includes weather-related items like 'coat' and 'umbrella' if used in relation to the weather. 


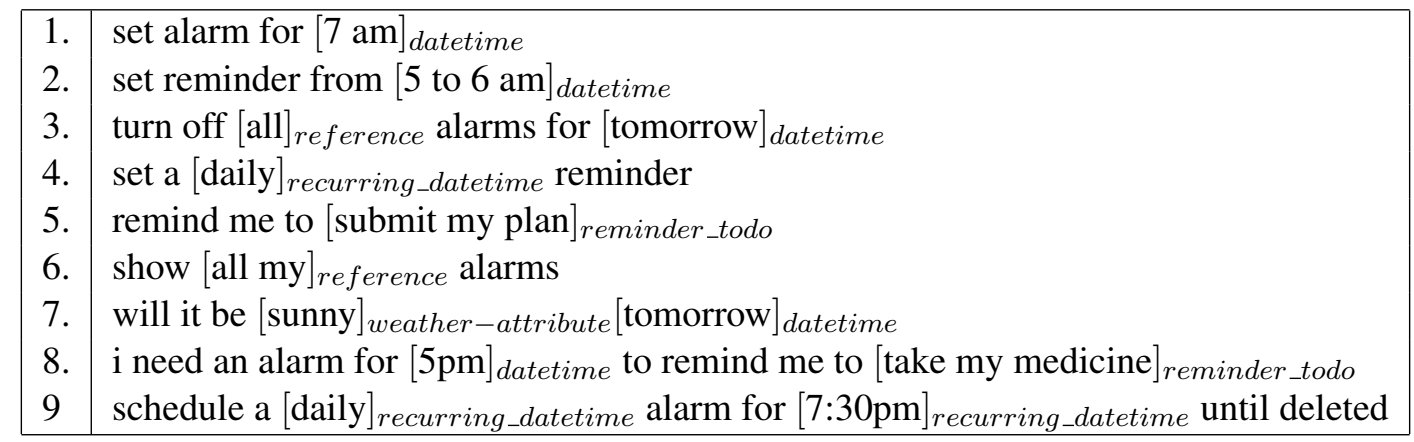

Figure 7: Example annotation for the slots. Slot-spans are indicated by square brackets and their label is shown directly behind the span.

English:

Remind me to call mom today at 2 p.m.

German:

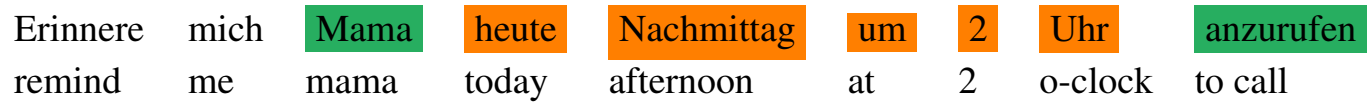

Figure 8: Example of sentence-final verb in German. Green: reminder_todo, Orange: datetime

\subsection{Language-specific exceptions}

German/Dutch sentence-final verbs and split phrases German and Dutch allow for sentencefinal verbs. For example, reminder todo slots might end up being split in the German translation, as another phrase or slot might be in between. See for an example Figure 8. We annotate the resulting non-continuous span for 'call mum' as two separate reminder_todo entities.

Handling of difficulties in Arabic compound morphology We encountered three special morphological cases during translating and annotating Arabic samples, in which a word can be a compound of two segments:

1. Verbs can be prefixed by a preposition (e.g. to, for, by):

ذكرني بشراء الحليب

Remind me to buy milk

In this case, we decided to annotate the whole compound word بشراء (EN: to buy milk) as a reminder.

2. Nouns can be suffixed with a possessive determiner (e.g. my, our):

اغلق منبهي

Turn off my alarm

In this case we decided to annotated the whole compound word منهي (EN: "my alarm") as reference-part. This label was only used for Arabic, and is converted to reference during evaluation.

3. Singular and dual nouns can be written as one word without the need to modify it with the numbers one or two:

$$
\begin{aligned}
& \text { two stars } \mapsto \text { نجمتين } \\
& \text { نne star } \mapsto \text { نممة }
\end{aligned}
$$

Serbian orthographic transcription of foreign named entities The rules of standard Serbian spelling dictate that named entities from other languages be transcribed orthographically into Serbian. Since Serbian orthography is near-perfectly phonemic, this means that the transcription of named entities will be highly dependent on the source pronunciation (although not solely determined by it). As a result, different transcription rules apply to not only different types of named entities but also different source languages.

When translating to Serbian, we recognize three groups of named entities that require different rules for translation and annotation:

1. Common named entities, such as well-known place names, names of historical and public figures, as well as names of popular literary, visual, and musical works. Such named entities are simply translated into their established 
Serbian equivalents.

2. Less common named entities, such as lesserknown place names or names of public figures. When translating these entities, we follow the pronunciation and transcription rules specific to the source language, which means that we have to look up the origin and native pronunciation of all unfamiliar entities. However, since many of these are unlikely to be found in Serbian texts or corpora, we keep the source transcription (in English) in square brackets for future reference. We annotate the source and its Serbian translation as two separate spans with the same label.

3. Names of songs, playlists, video games, and lesser-known films and TV programs. These were neither translated nor transcribed, but left as they are in the source text, as such named entities are not commonly transcribed and have no official translation in Serbian. 